\title{
West Bank
}

National Cancer Institute

\section{Source}

National Cancer Institute. West Bank. NCI Thesaurus. Code C123774.

5860 square kilometers in the Middle east that is west of Jordan and east of Israel. 